\title{
Dietas populares: Dieta cetogénica ${ }^{1}$
}

\author{
Kelsey Gemmill, Daniela Rivero-Mendoza, y Wendy Dahl²
}

La dieta cetogénica es una dieta alta en grasas y muy baja en carbohidratos. El primer uso documentado de la dieta cetogénica fue en 1921 para tratar la epilepsia en niños (Kim 2017). En los últimos años, la dieta cetogénica ha ganado un resurgimiento como un medio potencial para perder peso (Harvey, Holcomb, and Kolwicz 2019). La dieta cetogénica se ha vuelto popular debido al respaldo de las celebridades y las influencias de las redes sociales, pero $i$ es segura y efectiva?

\section{¿Qué es la dieta cetogénica?}

La dieta cetogénica promueve un consumo alto de grasas, hasta el 90\% de la energía total, proteínas moderadas (1 $\mathrm{g} / \mathrm{kg}$ de peso corporal / día) y un consumo muy bajo de carbohidratos: menos de 50 g por día (Veyrat-Durebex et al. 2018). La dieta cetogénica clásica es una dieta 4:1, que proporciona 4 gramos de grasa por cada 1 gramo de proteína y carbohidratos combinados (Fedorovich, Voronina, and Waseem 2018). En el tratamiento de la epilepsia, la proporción puede variar y proporcionar menos grasa (por ejemplo, 3,75:1). Una dieta cetogénica puede ser isocalórica, lo que significa que proporciona las calorías adecuadas para mantener el peso corporal, o baja en calorías y muy baja en calorías, que si se sigue, daría como resultado la pérdida de peso (Trimboli et al. 2020). Una dieta alta en grasas y muy baja en carbohidratos forzará al cuerpo a la cetosis, un estado metabólico en el que la grasa proporciona la mayor parte del combustible (Veyrat-Durebex et al. 2018).

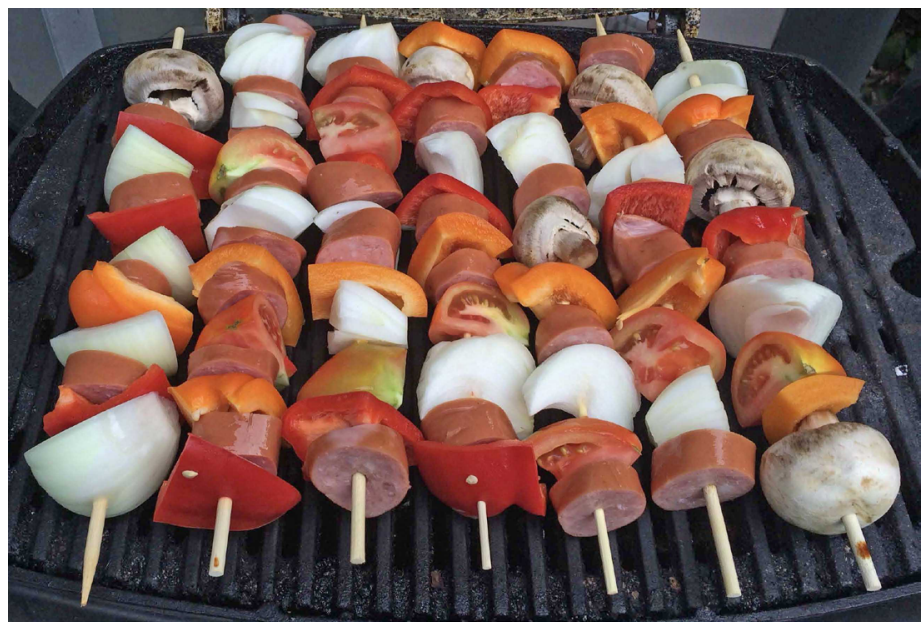

Existe una diferencia entre la cetosis, que es el resultado de la dieta cetogénica o la inanición (periodo de hambruna), y la cetoacidosis diabética. Las personas sanas en cetosis pueden usar cetonas para obtener energía y los niveles de cetonas no alcanzan un nivel no saludable en la sangre (Fedorovich, Voronina, and Waseem 2018). La cetoacidosis diabética ocurre en personas con diabetes cuando la glucosa en sangre se vuelve muy alta pero no se puede absorber ni utilizar debido a la falta de insulina (Fedorovich, Voronina, and Waseem 2018). La grasa se descompone para obtener energía y las cetonas se acumulan en la sangre a niveles muy peligrosos (Kanikarla-Marie and Jain 2016). La cetoacidosis diabética es una afección médica grave que puede provocar coma y la muerte (Kanikarla-Marie and Jain 2016) y no debe confundirse con la cetosis.

\section{¿Qué es la cetosis?}

En circunstancias normales, el cuerpo humano utiliza preferiblemente glucosa como energía (Fedorovich,

1. Este documento, FSHN20-44s, es uno de una serie de publicaciones del Food Science and Human Nutrition, Servicio de Extensión Cooperativa de la Florida, Instituto de Alimentos y Ciencias Agrícolas, Universidad de la Florida. (UF/IFAS). Fecha de primera publicación: octubre 2020. Visite nuestro sitio web EDIS en https://edis.ifas.ufl.edu.

2. Kelsey Gemmill, former MS-DI student; Daniela Rivero-Mendoza, Extension and research coordinator; and Wendy Dahl, associate professor, Food Science and Human Nutrition Department, UF/IFAS Extension; Gainesville, FL 32611.

The Institute of Food and Agricultural Sciences (IFAS) is an Equal Opportunity Institution authorized to provide research, educational information and other services

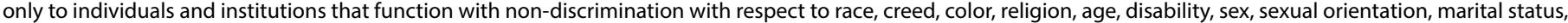

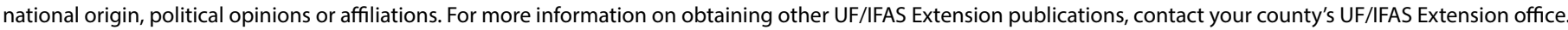
U.S. Department of Agriculture, UF/IFAS Extension Service, University of Florida, IFAS, Florida A \& M University Cooperative Extension Program, and Boards of County Commissioners Cooperating. Nick T. Place, dean for UF/IFAS Extension. 
Voronina, and Waseem 2018). Una vez que los alimentos que contienen carbohidratos se consumen y digieren, los carbohidratos se absorben como azúcar, principalmente glucosa. La cantidad diaria recomendada (RDA, por sus siglas en inglés) de carbohidratos es de 130 gramos por día para adultos, que se cree que es adecuada para el suministro de glucosa al cerebro y al sistema nervioso (Institute of Medicine, Food and Nutrition Board 2005). Sin embargo, en determinadas circunstancias, como la inanición (periodo de hambruna) o la ingesta muy baja de carbohidratos, no hay suficiente glucosa para dar al cuerpo la energía que necesita (Fedorovich, Voronina, and Waseem 2018). En cambio, el cuerpo humano tiene la capacidad de adaptarse a estos cambios y generar energía utilizable en forma de cuerpos cetónicos a partir de la grasa (Fedorovich, Voronina, and Waseem 2018). Cuando el cuerpo depende de los cuerpos cetónicos para obtener energía, se encuentra en un estado de "cetosis" (Fedorovich, Voronina, and Waseem 2018). Durante el ayuno o al consumir una dieta cetogénica, las cetonas se pueden usar para obtener energía (Fedorovich, Voronina, and Waseem 2018).

\section{¿Es la dieta cetogénica lo mismo que la dieta Atkins?} Tanto la dieta cetogénica como la de Atkins restringen la ingesta de carbohidratos. Sin embargo, la dieta cetogénica también restringe la ingesta de proteínas, solo lo suficiente para satisfacer las necesidades, mientras que la dieta Atkins no restringe las proteínas.

\section{¿Qué hay en el menú de una dieta cetogénica?}

Los alimentos que contienen poco o ningún carbohidrato, como carnes, aves, pescado, huevos, queso, vegetales de hojas verdes, nueces, aceite, mantequilla, mayonesa y crema (USDA 2020), se consumen comúnmente en una dieta cetogénica. Aunque las grasas insaturadas, que se consideran saludables, están permitidas en la dieta cetogénica, las grasas saturadas, asociadas con el riesgo cardiovascular, también se recomiendan en este patrón dietético. Se pueden utilizar endulzantes no nutritivos. Los alimentos que deben evitarse debido a su mayor contenido de carbohidratos incluyen pan, cereales, frutas, verduras con almidón, productos horneados y legumbres (por ejemplo, frijoles, lentejas y guisantes) (USDA 2020). Por lo general, las verduras se limitan a vegetales de hoja verde, como el brócoli, coliflor, pimientos, cebollas, pepinos, apio y otros vegetales bajos en carbohidratos. Las frutas están mucho más restringidas debido a su mayor contenido de carbohidratos, en comparación con las verduras sin almidón. Es importante recordar que muchos de los alimentos restringidos en la dieta cetogénica son los alimentos que mejoran la salud, recomendados por las Guías Alimentarias para los Estadounidenses, como los cereales integrales, las frutas, las verduras y los productos lácteos bajos en grasa (USDA 2015).

\section{¿Cuáles son los posibles beneficios para la salud de una dieta cetogénica?}

La dieta cetogénica es beneficiosa para el tratamiento de algunos síndromes epilépticos intratables (trastornos convulsivos) (D’Andrea Meira et al. 2019). Actualmente se están estudiando sus posibles efectos beneficiosos en adultos con obesidad, diabetes y enfermedades neurológicas. En un estudio de adultos con sobrepeso y diabetes tipo 2, la dieta cetogénica, durante 32 semanas, mejoró el control glucémico y resultó en una mayor pérdida de peso en comparación con un programa de alimentación bajo en grasas para la diabetes (Saslow et al. 2017). La mayoría de los estudios de la dieta cetogénica y los resultados relacionados con las enfermedades neurológicas se han realizado en animales de laboratorio (McDonald and Cervenka 2018) $y$, por lo tanto, los resultados no se pueden aplicar a los seres humanos.

¿Cómo podría la dieta cetogénica facilitar la pérdida de peso? Una teoría está relacionada con el efecto saciante de la grasa, lo que sugiere que una dieta alta en grasas reduce el apetito y, por lo tanto, disminuye el consumo general de alimentos. Sin embargo, los resultados de los estudios que analizan los efectos saciantes de la grasa han sugerido recientemente lo contrario (Warrilow et al. 2019). Otro mecanismo propuesto es que las dietas cetogénicas mantienen el gasto de energía habitual en reposo: la cantidad de energía gastada en funciones fisiológicas como la respiración, la función cerebral y el latido del corazón (Gershuni, Yan, and Medici 2018). Con frecuencia, la pérdida de peso provoca una disminución del gasto de energia en reposo, lo que, a su vez, facilita la recuperación de peso. Al seguir una dieta cetogénica que proporciona menos calorías de las que se gastan, el cuerpo quemará más grasa para obtener energía de la que acumulará, lo que puede conducir a una mayor pérdida de masa grasa mientras se mantiene la masa magra (Gershuni, Yan, and Medici 2018). Se necesita más investigación para confirmar los efectos metabólicos de una dieta cetogénica.

Como las grasas tienen un alto contenido energético ( 9 frente a 4 calorías por gramo de proteínas o carbohidratos), se debe tener cuidado de no consumir calorías en exceso, ya que esto conducirá a un aumento de peso. Para mantener la ingesta de calorías bajo control, los alimentos ricos en grasas y densos en energía deben limitarse a porciones 
pequeñas. Se recomienda consultar con un nutricionista dietista registrado para planificar adecuadamente las dietas cetogénicas para el mantenimiento o la pérdida de peso.

\section{¿Cuáles son los riesgos de una dieta cetogénica?}

La dieta estadounidense típica consumida por los adultos es mucho más alta en carbohidratos $(46 \%-48 \%$ de la energía total) y más baja en grasas (34\%-35\% de la energía total) que la dieta cetogénica (National Center for Health Statistics, Centers for Disease Control and Prevention 2017). Debido a las restricciones en la ingesta de cereales integrales, frutas y verduras, la dieta cetogénica suele ser muy baja en fibra dietética y ciertas vitaminas y minerales que se encuentran en los cereales enriquecidos y los productos frescos (Gordon 2019). Seguir la dieta cetogénica, en comparación con una dieta alta en carbohidratos, conducirá a cambios en el consumo de nutrientes y puede conducir a deficiencias de nutrientes a largo plazo (McSwiney and Doyle 2019). El estreñimiento es una queja frecuente de la dieta cetogénica porque esta dieta restringe alimentos ricos en fibra como cereales integrales, frutas y algunas verduras.

La dieta cetogénica puede tener efectos negativos para la salud y el bienestar. A corto plazo, algunas personas que siguen una dieta cetogénica han informado síntomas similares a los de la gripe, que incluyen dolor de cabeza, fatiga, náuseas, mareos, trastornos gastrointestinales, disminución de la energía, entre otros (Bostock et al. 2020). Se ha informado letargo, deshidratación, acidosis, cambios de humor y aumento de infecciones, vómitos y estreñimiento durante los primeros días de uso de la dieta cetogénica en algunos niños con epilepsia (Vining et al. 1998).

A largo plazo, una dieta cetogénica puede contribuir a la aparición de enfermedades cardiovasculares debido a su alto contenido de grasas saturadas. En las Guías Alimentarias para los Estadounidenses, se recomienda consumir menos del 10 por ciento de las calorías de grasas saturadas (mantecas), ya que reemplazar las grasas saturadas (manteca) con grasas insaturadas (aceites) puede reducir el riesgo de enfermedad cardiovascular (USDA 2015). Es muy poco probable que se pueda lograr esta recomendación siguiendo una dieta cetogénica. Además, debido a su alto contenido de grasa, la dieta cetogénica no se recomienda para personas con ciertas afecciones hepáticas, enfermedad de la vesícula biliar o aquellas a las que se les extirpó la vesícula biliar, enfermedad pancreática y problemas de tiroides (Gordon 2019). Los informes han descrito el desarrollo de cálculos renales (piedra en el riñon) en niños con una dieta cetogénica (Choi et al. 2010).

\section{Resumen}

La dieta cetogénica es una dieta muy alta en grasas y baja en carbohidratos. Seguir una dieta cetogénica induce a un estado de cetosis, donde la grasa se quema como principal fuente de energía. Si bien la dieta cetogénica puede ser beneficiosa como parte del tratamiento médico de las personas afectadas por síndromes epilépticos, no se ha demostrado su eficacia para la pérdida de peso a largo plazo. Tampoco para el tratamiento de enfermedades neurológicas y sobre todo sus riesgos, especialmente a largo plazo, no están claros. A corto plazo, una dieta cetogénica restringida en calorías puede conducir a una pérdida de peso similar a otras dietas restrictivas. En general, los riesgos de la dieta cetogénica pueden superar los posibles beneficios para la salud. Hable con su proveedor de atención médica antes de adoptar una dieta cetogénica.

\section{Referencias}

Bostock, E. C. S., K. C. Kirkby, B. V. Taylor, and J. A. Hawrelak. 2020. 'Consumer Reports of 'Keto Flu' Associated with the Ketogenic Diet." Frontiers in Nutrition 7(20). https://doi.org/10.3389/fnut.2020.00020

Choi, J. N., J. E. Song, J. I. Shin, H. D. Kim, M. J. Kim, and J. S. Lee. 2010. "Renal Stone Associated with the Ketogenic Diet in a 5-Year-Old Girl with Intractable Epilepsy." Yonsei Medical Journal 51 (3): 457-9.

D’Andrea Meira, I., T. T. Romão, H. J. Pires do Prado, L. T. Krüger, M. E. P. Pires, and P. O. da Conceição. 2019. "Ketogenic Diet and Epilepsy: What We Know So Far." Frontiers in Neuroscience 13:5.

Fedorovich, S. V., P. P. Voronina, and T. V. Waseem. 2018. "Ketogenic Diet versus Ketoacidosis: What Determines the Influence of Ketone Bodies on Neurons?" Neural Regeneration Research 13 (12): 2060-3.

Gershuni, V. M., S. L. Yan, and V. Medici. 2018. "Nutritional Ketosis for Weight Management and Reversal of Metabolic Syndrome." Current Nutrition Reports 7 (3): 97-106.

Gordon, B. 2019. "What Is the Ketogenic Diet?" https:// www.eatright.org/health/weight-loss/fad-diets/what-is-theketogenic-diet [Accessed 24th July 2020]. 
Harvey, K. L., L. E. Holcomb, and S. C. Kolwicz. 2019. "Ketogenic Diets and Exercise Performance." Nutrients 11 (10): 2296.

Institute of Medicine, Food and Nutrition Board. 2005. Dietary Reference Intakes for Energy, Carbohydrate, Fiber, Fat, Fatty Acids, Cholesterol, Protein, and Amino Acids. Washington, D.C.: The National Academies Press.

Kanikarla-Marie, P., and S. K. Jain. 2016. "Hyperketonemia and Ketosis Increase the Risk of Complications in Type 1 Diabetes." Free Radical Biology and Medicine 95:268-77.

Kim, J-M. 2017. "Ketogenic Diet: Old Treatment, New Beginning." Clinical Neurophysiology Practice 2:161-2.

McDonald, T. J. W., and M. C. Cervenka. 2018. "Ketogenic Diets for Adult Neurological Disorders." Neurotherapeutics 15 (4): 1018-31.

McSwiney, F. T., and L. Doyle. 2019. "Low-Carbohydrate Ketogenic Diets in Male Endurance Athletes Demonstrate Different Micronutrient Contents and Changes in Corpuscular Haemoglobin over 12 Weeks." Sports (Basel) 7 (9). https://www.mdpi.com/2075-4663/7/9/201

National Center for Health Statistics, Centers for Disease Control and Prevention. 2019. "Dietary Intake for Adults Aged 20 and Over." https://www.cdc.gov/nchs/fastats/diet. htm [Accessed 24th July 2020].

Saslow, L. R., A. E. Mason, S. Kim, V. Goldman, R. PloutzSnyder, H. Bayandorian et al. 2017. "An Online Intervention Comparing a Very Low-Carbohydrate Ketogenic Diet and Lifestyle Recommendations versus a Plate Method Diet in Overweight Individuals with Type 2 Diabetes: A Randomized Controlled Trial." Journal of Medical Internet Research 19 (2): e36.

Trimboli, P., M. Castellana, D. Bellido, and F. F. Casanueva. 2020. "Confusion in the Nomenclature of Ketogenic Diets Blurs Evidence." Reviews in Endocrine and Metabolic Disorders. 21 (1): 1-3.

United States Department of Agriculture (USDA). 2015. Dietary Guidelines for Americans 2015-2020. https:// health.gov/our-work/food-nutrition/2015-2020-dietaryguidelines/guidelines/ [Accessed 24th July 2020].

United States Department of Agriculture. 2020. FoodData Central. https://fdc.nal.usda.gov/index.html [Accessed 24th July 2020].
Veyrat-Durebex, C., P. Reynier, V. Procaccio, R. Hergesheimer, P. Corcia, C. R. Andres et al. 2018. "How Can a Ketogenic Diet Improve Motor Function?” Frontiers in Molecular Neuroscience 11 (15). https://doi.org/10.3389/ fnmol.2018.00015

Vining, E. P. G., J. M. Freeman, K. Ballaban-Gil, C. S. Camfield, P. R. Camfield, G. L. Holmes et al. 1998. "A Multicenter Study of the Efficacy of the Ketogenic Diet." Archives of Neurology 55 (11): 1433-7.

Warrilow, A., D. Mellor, A. McKune, and K. Pumpa. 2019. "Dietary Fat, Fibre, Satiation, and Satiety-a Systematic Review of Acute Studies." European Journal of Clinical Nutrition 73 (3): 333-44. 